\title{
A Single Crystal Niobium RF Cavity of the TESLA Shape
}

\author{
W. Singer, X. Singer, P. Kneisel \\ DESY, Notkestrasse 85, D-22607 Hamburg, Germany \\ Thomas Jefferson National Accelerator Facility, Newport News, Virginia 23606, USA
}

\begin{abstract}
A fabrication method for single crystal niobium cavities of the TESLA shape was proposed on the basis of metallographic investigations and electron beam welding tests on niobium single crystals. These tests showed that a cavity can be produced without grain boundaries even in the welding area. An appropriate annealing allows the outgassing of hydrogen and stress relaxation of the material without destruction of the single crystal. A prototype single crystal single cell cavity was build. An accelerating gradient of $37.5 \mathrm{MV} / \mathrm{m}$ was reached after approximately $110 \mu \mathrm{m}$ of Buffered Chemical Polishing (BCP) and in situ baking at $120^{\circ} \mathrm{C}$ for 6 hrs with a quality factor exceeding $2 \times 1010$ at $1.8 \mathrm{~K}$. The developed fabrication method can be extended to fabrication of multi cell cavities.
\end{abstract}

Keywords: Cavity, single crystal, accelerating gradient, material properties.

PACS: 29.17.+w, 85.25.-j, 65.40.-b

\section{INTRODUCTION}

There are theoretical and experimental claims that grain boundaries GBs reduce the thermal break down limit of an rf cavity (see for example [1-3]). Some of causes are listed below.

GBs are responsible for magnetic field enhancement (steps on GBs after BCP)

GBs enhance the possibility of penetration of external magnetic field (GBs are planar weak links with reduced critical current density)

GBs add to the RF resistance due to vortices penetrating along the grain boundary (reduce the quality factor Qo)

GBs enhance the possibility of hydrogen absorption and diffusion

GBs are the areas that gathered impurities (reduced RRR)

GBs reduce the thermal conductivity at low temperatures (reduce the phonon contribution)

GBs enhance the surface roughness

A single crystal cavity with no grain boundaries has definitely a potential to improve the cavity performance substantially because by nature it does not contain any

\author{
CP927, Single Crystal-Large Grain Niobium Technology, International Niobium Workshop \\ edited by G. R. Myneni, T. Carneiro, and A. Hutton \\ (C) 2007 American Institute of Physics 978-0-7354-0437-3/07/\$23.00
}


interruption of the crystal lattice orientation. In addition, one can expect a much easier surface preparation procedure for the superconducting surface. The removal of the surface damage layer from the inner surface - necessary for good cavity performance - can be done by standard BCP 1:1:2, because there is no excessive grain etching or different etching speed of different oriented grains. A very smooth surface can be easily produced.

Very high potential of large grain and single crystal cavities was recently demonstrated at JLab [4-7]. An accelerating gradient of Eacc 46 MV/m was measured on a $2.3 \mathrm{GHz}$ cavity (Low Loss LL shape) fabricated from two single crystal discs of approximately 160 mm diameter.

For an ILC or XFEL cavity shape (TESLA, LL or Reentrant RE) a single crystal disc of $270 \mathrm{~mm}$ diameter is required. It seems that in the short term industry will not be in position to produce single crystal niobium of such dimensions.

Therefore, at DESY investigations were conducted to find a method to enlarge smaller single crystal high purity niobium, - available on the market- without breaking-up the single crystal to the desire diameter and a fabrication method for a TESLA like single crystal cavity was proposed.

\section{FABRICATION}

The following aspects have been investigated on samples and taken into consideration for the fabrication proposal.

- Definite enlargement of the single crystal disc diameter is possible without destroying the single crystal structure.

- The single crystals keep the crystallographic structure and after forming of the cavity half cell from a disc by deep drawing the orientation perpendicular to the surface remains.

- Appropriate heat treatment will not destroy the deformed single crystal

- Two single crystals will grow together by EB welding, if the orientation of the crystals is matched.

Illustrations of these aspects are shown below in figures 1 to 4 , where the dependence of the microstructure on the degree of deformation before and after appropriate heat treatment is depicted.

The aim of the heat treatment is twofold: removing hydrogen from the lattice and to stress relieve the material - required for good cavity performance- without destroying the single crystal. The appropriate heat treatment depends on the degree of deformation. The degree of deformation of available single crystals of high purity niobium is expected to be about $20-70 \%$.

The following heat treatments were applied: annealing at $400-500^{\circ} \mathrm{C}$ for $2-6$ hours as a first step and annealing at $750-850^{\circ} \mathrm{C}$ for $1-3 \mathrm{~h}$ as second step. The first step is applied to recover the material (to eliminate possible nuclei, which could create new crystals), the second step provides the outgassing of hydrogen and stress relaxation of the cavity. 
The deformation was done by rolling; the degree of deformation was $60-90 \%$. The metallographic analysis of the surface perpendicular to the rolling direction (Fig.1-4) shows that, if the deformation degree is smaller as $70 \%$, the single crystals will not be destroyed; the microstructure of the deformed samples after annealing is similar to to that of the original single crystal. If the critical deformation degree of $70 \%$ is exceeded, recrystallization takes place during annealing. As can be seen in figures 3-4, new small crystals appear and as expected the new crystals become smaller in size as the degree of deformation increases

As these preliminary investigations show, it seems to be possible to enlarge a smaller niobium single crystal from the inside of an ingot to a disc of the dimensions needed for the deep drawing or spinning of a half cell for a TESLA type cavity. In addition the experiments have shown that the crystallographic structure and the crystal orientation perpendicular to the surface remain after deep drawing of the disc into a half cell as expected due to the rather small degree of deformation (less than 10-15\%) produced during deep drawing.

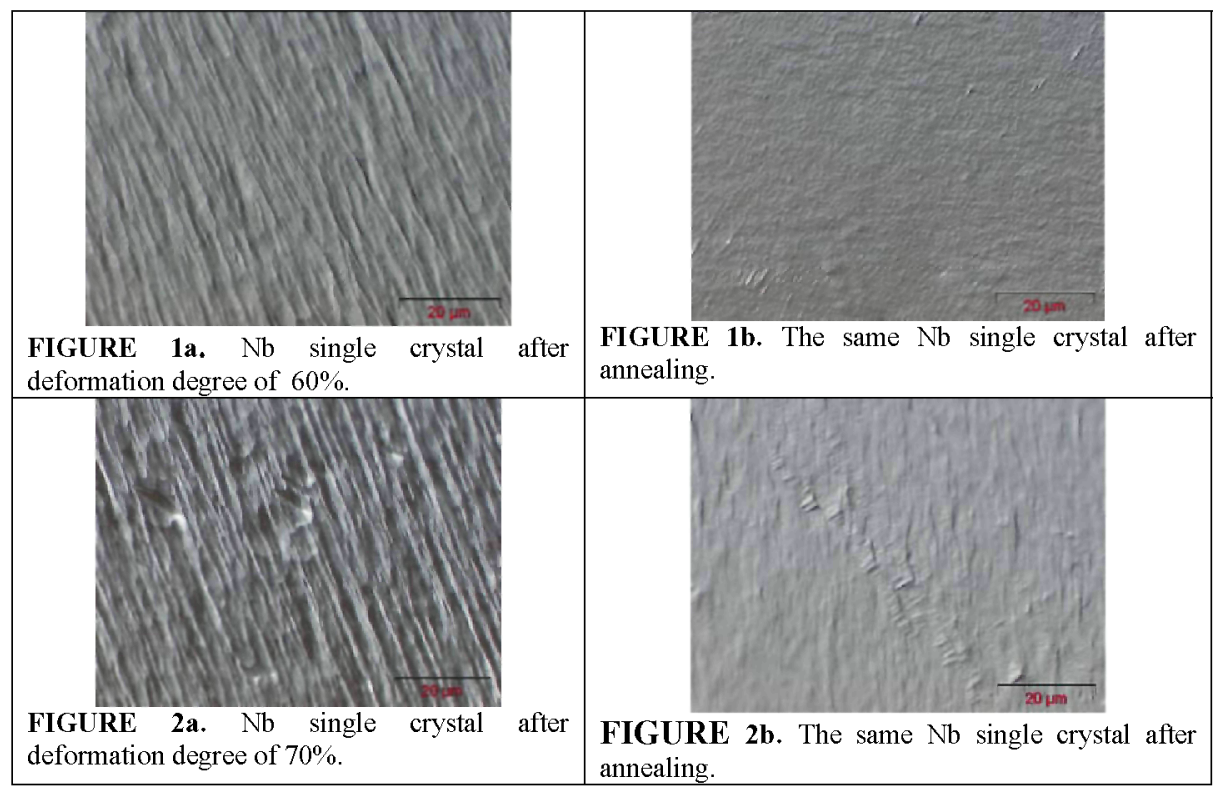




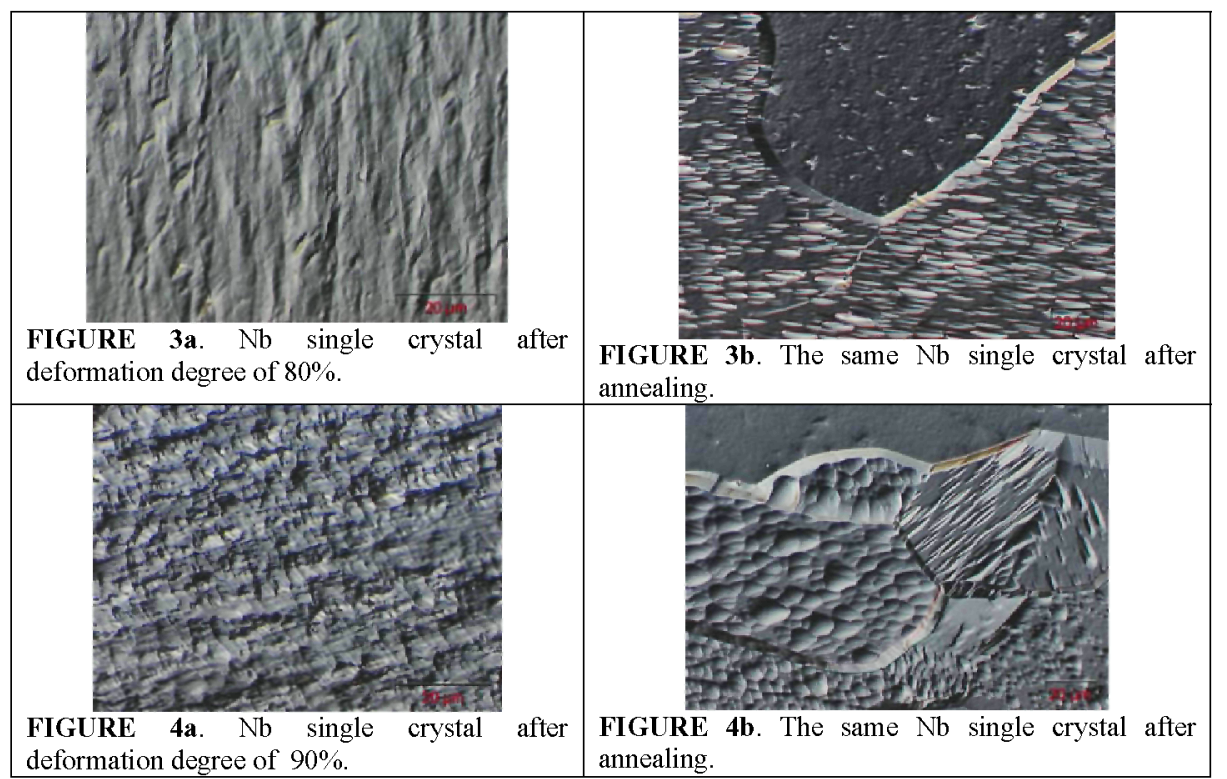

Further investigations focused on the issues of electron beam welding of single crystals. It turned out that two single crystals will grow into one single crystal, if the crystallographic orientations are matched at the EB seam.

The proposed electron beam welding procedure was proven on a single crystal sample. The results of metallographic analysis of the cross section can be seen in fig. $5 \mathrm{a}$, b: whereas unmatched orientations produce a pronounced grain boundary (Fig. 5a), matched orientations of both single crystals grow together without interface (grain boundary free) (Fig. 5b).

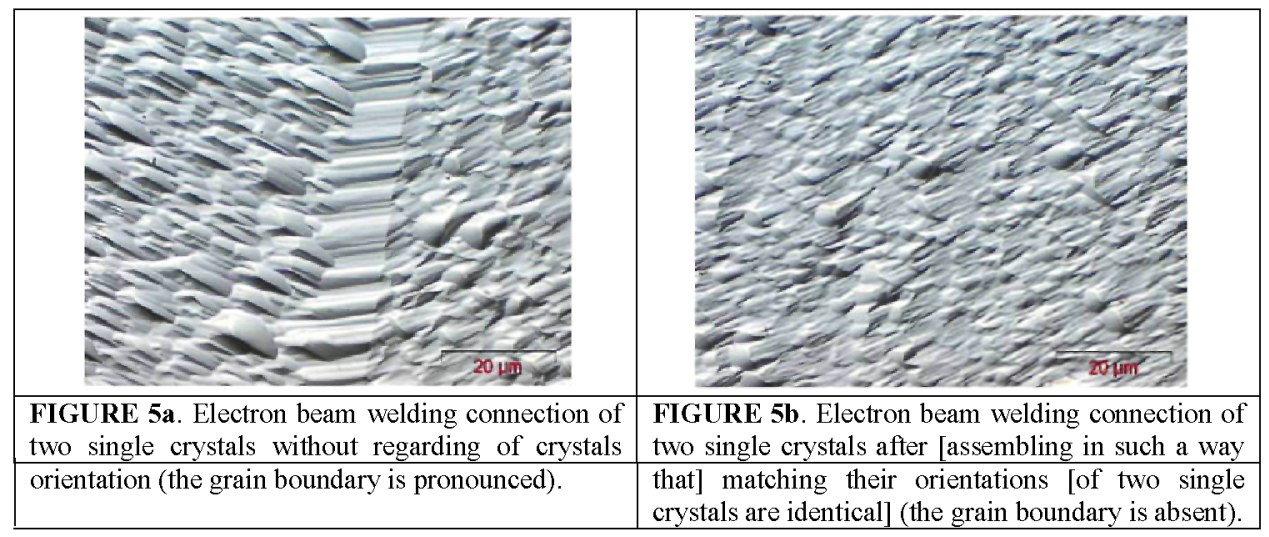


The orientations of the two single crystal samples before and after EB (Fig. 5b) were determined by means of Laue back scattering. As figure 6 demonstrates, the same crystallographic orientations of both primary samples (left and write) were preserved after EB.

Based on the investigations described above, a prototype single crystal cavity of the TESLA shape was produced. A niobium ingot supplied by W.C. HERAEUS with a single crystal of approximately $150 \mathrm{~mm}$ in diameter in its center was used. Two discs cut from the ingot were marked to keep track of the orientation of the single crystals during the production steps to the half cells and of the cavity. The diameters of the discs were increased by rolling at RWTH Aachen. Deep drawing of the half cells and the electron beam welding was done at ACCEL. Prior to electron beam welding the half cells were assembled in such a way that the crystal orientations were identical to the two discs before cutting.

The crystal pattern of the original W.C. HERAEUS ingot with the large central single crystal and the single cell single crystal cavity are shown in Fig. 7 and Fig. 8, respectively. The cell surface is very shiny after only $20 \mu \mathrm{m}$ of chemical polishing in contrast to the dull appearance of the cut off tubes produced from conventional polycry stalline niobium; no grain boundaries are pronounced at the welding area.

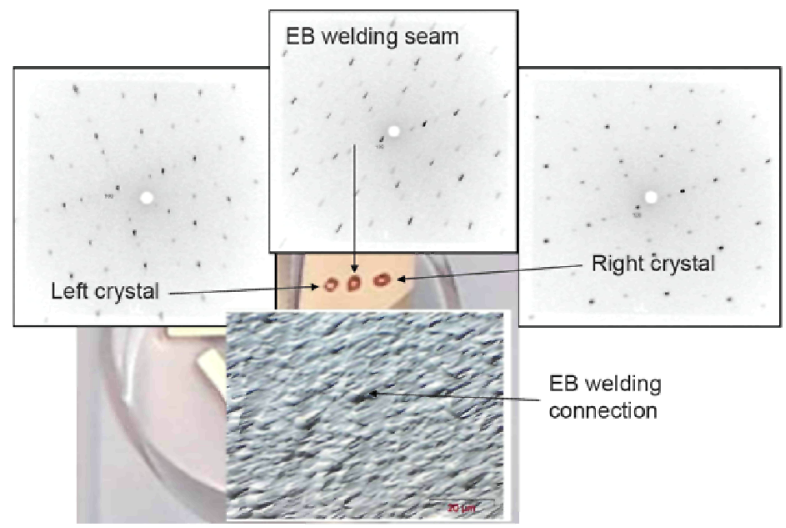

FIGURE 6. Laue back scattering X-Ray reflexes (the same in both welded together crystals and in the welding seam). 


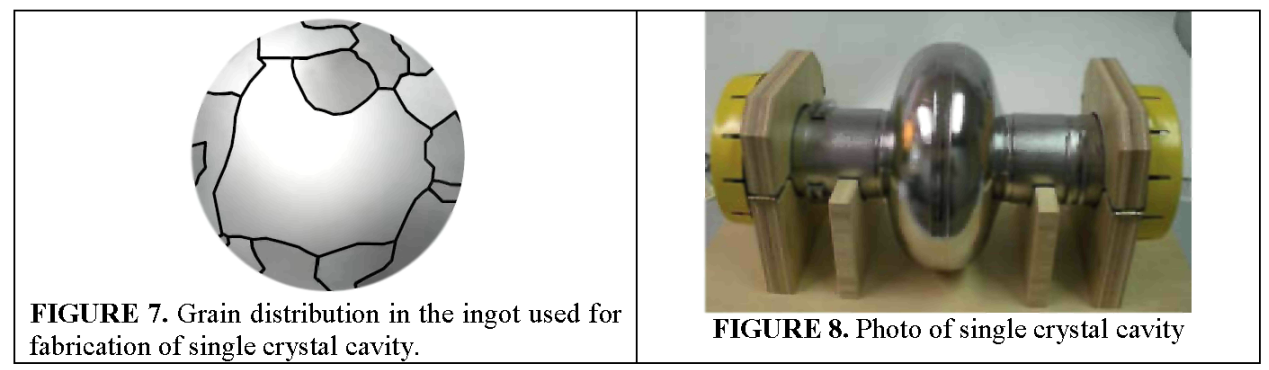

\section{TREATMENT AND RF TESTS}

Surface treatment and a series of RF tests after successive material removal with BCP were done at JLab. A best accelerating gradient of $37.5 \mathrm{MV} / \mathrm{m}$ was reached after only 112 $\mu \mathrm{m} \mathrm{BCP}$ and in situ baking at $120^{\circ} \mathrm{C}$ for $6 \mathrm{hrs}$ with the quality factor of $2 \times 1010$ at $1.8 \mathrm{~K}$ (Fig. 9).

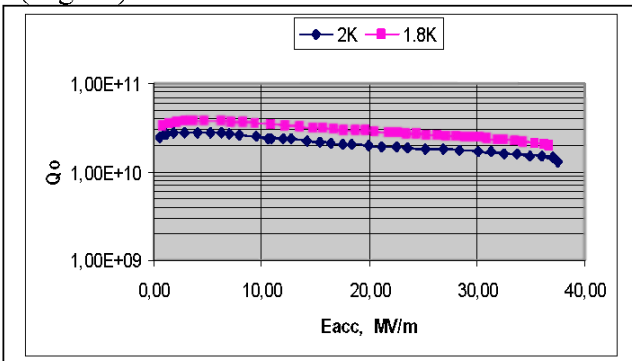

FIGURE 9. $Q($ Eace) curve for the single crystal single cell cavity after $112 \mu \mathrm{m} \mathrm{BCP}$ and in situ baking $120^{\circ} \mathrm{C}$ for $6 \mathrm{hrs}$.

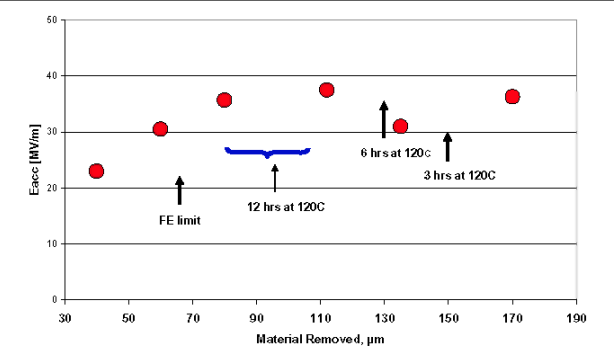

FIGURE 10. Eace vs. material removal on single crystal single cell cavity.

The limitation was caused by a quench. Fig. 10 shows the quench field as a function of the removed surface layer after each material removal step. It is known from experiments on fine grain material that heat treatment around $800^{\circ} \mathrm{C}$ provides for hydrogen outgassing and stress relaxation and in many cases an increased accelerating gradient could be obtained. Therefore, as a next step it is planned to anneal the cavity at an appropriate temperature, guided by annealing procedures developed on samples.

\section{OUTLOOK}

As shown above the first RF test results obtained with the single crystal single cell cavity are very encouraging. The single crystal option opens the possibility to achieve 
mirror like inner surfaces of a complete cavity cell by applying standard chemical treatment $\mathrm{BCP}(1: 1: 2)$. This may potentially lead to better superconducting parameters of the niobium and eventually to better cavity performance in terms of achievable accelerating field, quality factor and reproducibility of performance.

The developed method can be extended to fabrication of multi cell single crystal cavities by using the above described assembly method not only for equators but also for the irises welds.

A single crystal applied for cavity fabrication has a crystal orientation close to (100). In this connection appears the question, how the cavity performance can be influenced by the crystal orientation. This issue is not investigated up to now and has a definite potential. Anisotropy of the superconducting energetic gap $2 \Delta / \mathrm{Tc}$ in the $4 \mathrm{~d}$ and $5 \mathrm{~d}$ transition metals including $\mathrm{Nb}$ is well known /8/. As one of the main parameters of superconductivity $\Delta$ should influence the main physical properties of the material (for example Hc and surface resistance). Therefore, it is not inconceivable that fabrication of single crystal cavities with preferred orientation could lead to further improvement of performance.

Moreover one could speculate that cavities without grain boundaries should produce lower losses; resulting in higher Q-values. The results in fig. 9, were a rather high Qo up to $2 \times 10^{10}$ at maximal gradient and a temperature of $1.8 \mathrm{~K}$ is shown, seem to support this speculation; however, more statistics is needed.

It is hoped that with single crystal niobium cavities a better understanding of the underlying physics of limitations in RF cavities can be gained, since effects due to the presence of grain boundaries in poly-crystalline niobium such as grain boundary segregation, flux penetration or weak link behaviour are absent. This eventually should lead to simplified treatments and better reproducibility of cavity performances.

\section{ACKNOWLEDGMENTS}

We would like to express gratitude to Mr. K.-R. Baldner, J. Van Santen for the rolling of the single crystal discs, to M. Pekeler and J. Schwellenbach for the fruitful joint work in the single crystal cavity fabrication, to M. Spiwek for the sample characterization by Laue back scattering, to A. Schmidt for EB welding of samples and to R. Brinkmann, D. Proch, G. Rao Myneni and B. Spaniol for productive discussions.

\section{REFERENCES}

1. A. Gurevich, Pushing the Limits of RF Superconductivity workshop, ANL, Sept 22-24, 2004

2. P. J. Lee, A. A. Polyanskii, A. Gurevich, A. A. Squitieri, D. C. Larbalestier, P. C. Bauer, C. Boffo, H. T. Edwards. Proceedings of 12 th International Workshop on RF Superconductivity, Ithaca, USA, July $10-15,2005$

3. P. Bauer. Review of Models of RF Surface Resistance in High Gradient Niobium Cavities for Particle Accelerators. FNAL Report TD-04-014, June 2004, 37 p. 
4. G. R. Myneni. Physical and Mechanical Properties of Single and Large Crystal High-RRR Niobium. Proceedings of 12th International Workshop on RF Superconductivity (SRF 2005), July 10 - 15, Ithaca, USA

5. P. Kneisel, G. R. Myneni, G. Ciovati, J. Sekutowicz, T. Carneiro. Performance of Large Grain and Single Crystal Niobium Cavities. Proceedings of 12th International Workshop on RF Superconductivity (SRF 2005), July 10 - 15, Ithaca, USA

6. P. Kneisel, G. R. Myneni, G. Giovati, J. Sekutowicz, T. Carneiro. Preliminary results from single crystal and very large crystal niobium cavities. Proceeding of of 2005 Particle Accelerator Conference, Knoxville, Tennessee, USA, Mai 16-20, 2005

7. P. Kneisel. Latest development in superconducting RF structures for beta $=1$ particle acceleration. Proceedings of 10th biennial European Particle Accelerator Conference, EPAC'06, Edinburgh, Scotland, 26 - 30 June, 2006

8. S. Wonsovski, J. Isjumov, E. Kurmaev. Superconductivity of transition metals alloys and compounds. Moscow, Nauka, 1977, 384 p. 